\title{
Preparation and Purification of Single-Walled Carbon Nanotubes
}

\author{
Jinling Gao ${ }^{1 *}$, Yongfu Lian ${ }^{2}$, Xiaoxue Gao ${ }^{1}$, Dezhao Hua
}

${ }^{1}$ Collage of Science, Heilongjiang Bayi Agricultural University, Daqing, 163319, China

${ }^{2}$ Key Laboratory of Functional Inorganic Material Chemistry, Ministry of Education, School of Chemistry and Materials Science, Heilongjiang University, Harbin 150080, China

\begin{abstract}
DOI: $10.36348 /$ sijcms.2020.v03i02.001
| Received: 10.02.2020 | Accepted: 18.02.2020 | Published: 23.02.2020
\end{abstract}

*Corresponding author: Jinling Gao

\section{Abstract}

As a functional material, high-purity single-walled carbon nanotubes are widely used in basic science and nanotechnology, such as mechanics, thermology, electricity, etc. Single-walled carbon nanotubes prepared by arc discharge method have fewer defects and are more suitable for mechanical and electrical applications; therefore, arc discharge method was adopted to prepare single-walled carbon nanotubes in this paper. Firstly, single-walled carbon nanotubes were prepared by dc arc discharge. Then, single-walled carbon nanotubes were purified by physical and chemical synthesis, after burning, acidification and selective dissolution in organic solvent, single-wall carbon nanotubes with high purity were obtained. Single-walled carbon nanotubes which purified before and after was investigated by Uvvisible-near-infrared spectroscopy, resonance Raman spectroscopy and high-resolution transmission electron microscopy. Keywords: Single-walled carbon nanotubes; Uv-visible near infrared spectrum; Resonance Raman spectrum.

Copyright @ 2020: This is an open-access article distributed under the terms of the Creative Commons Attribution license which permits unrestricted use, distribution, and reproduction in any medium for non-commercial use (NonCommercial, or CC-BY-NC) provided the original author and source are credited.

\section{INTRODUCTION}

Because of the large aspect ratio, large specific surface area and strong mechanical properties of singlewall carbon nanotubes (SWNTs), the conductivity of polymer composites can be enhanced by SWNTs [1]. Because of its excellent electrical conductivity, it can be used in the fabrication of lasers [2], sensors [3] and nanocomposites [4]. Because of its hollow tubular structure, it can be used to store drugs [5]. It can be seen that SWNTs are widely used in many fields, so the preparation of SWNTs is a significant work.

At present, there are many kinds of preparation methods of SWNTs [6], such as the dc arc discharge method, chemical vapor deposition and laser ignition method, the arc discharge method and laser ignition method need higher temperature, and the preparation of the SWNTs quality is higher, but the output of SWNTs prepared by laser ablation is low, the cost is higher, so it is not conducive to mass production and application. Chemical vapor deposition requires lower temperature and has the advantage of lower energy consumption and higher yield. However, SWNTs produced by this method under lower temperature have great structural defects, while SWNTs prepared by arc discharge method have fewer defects and are more suitable for mechanical and electrical applications. Therefore, this paper adopts arc discharge method to prepare SWNTs.

No matter which method is used to prepare SWNTs, amorphous carbon, residual metal catalyst particles, nano-carbon particles and other by-products will be generated. The presence of these impurities has a great impact on the physical and chemical properties of SWNTs. Therefore, it is great significance to purify SWNTs before it is applied. After purification, most of the amorphous carbon, catalyst residue, carbon nanoparticles and other by-products embedded in SWNTs can be effectively removed, and high-purity SWNTs are obtained, whose excellent physical and chemical properties can be fully reflected. According to the morphology of growth, process of growth, and catalysts used in the synthesis of nanotubes, many physical, chemical, or a combination of the two methods for purifying nanotubes have been reported in the literature [7]. The physical method is mainly used to disperse and centrifuge the products. The disadvantage is that the purification effect is poor and the advantage is that it is not destructive to the carbon tube. Chemical method is mainly through concentrated acid acidification samples, the disadvantage is the carbon tube structure has some destructive, the advantage is the purification effect is better. In this paper, large diameter 
SWNTs were prepared by arc discharge and purified by physical and chemical synthesis.

\section{MATERIALS AND METHODS Materials and reagents}

The toner and graphite rod are spectrum pure, the concentrated hydrochloric acid and ferrous sulfide are analytically pure, the yttrium nickel alloy and nickel powder are chemically pure, and the high purity nitrogen is $99.99 \%$.

\section{Instruments and equipment}

Low-speed centrifuge(LD4-2, the company of Beijing medical centrifuge), table centrifuge(TGL-16G, the company of Shanghai an ting scientific instrument), supercentrifuge(CP100WX, HITACHI of Japan), vacuum drying oven(DGF-6030, the company of Shanghai yiheng scientific instrument), electronic analytical balance(ALC-310.2, LTD. co. Beijing taike instrument), circulating water multi-purpose vacuum pump(SHB-IIIA, LTD. co. zhengzhou Great Wall science and trade), constant temperature magnetic stirring water bath(HJ-A2, LTD. co. changzhou maikonuo instrument), Uv-visible spectrophotometer (Uv-3600, HITACHI, Japan), micro laser Raman spectrometer(HR800, JY, France), transmission electron microscope(JEM2100Plus, Japan electron).

\section{METHODS}

\section{The preparation of SWNTs}

According to the method in reference [8], large diameter SWNTs was prepared by dc arc discharge. A spectrum pure hollow cylindrical graphite rod with an inner diameter of $6 \mathrm{~mm}$ and an inner length of $120 \mathrm{~mm}$ was used as the anode, which was equipped with a uniform mixture of growth promoter $\mathrm{Fe} \mathrm{S}$, Ni powder was as metal catalyst, alloy $\mathrm{YNi}_{2}$ was as catalyst and spectrum pure carbon powder was as the raw materials. The mass percentage of each component is 10, 10, 30 and $50 \%$, respectively. The cathode is a cylindrical graphite block with a base radius of $2 \mathrm{~cm}$ and a height of $2 \mathrm{~cm}$. The helium pressure was adjusted to $480 \mathrm{~T}$ and the current intensity was 100 A. During the whole arc discharge, the distance between the anode and cathode remained about $0.5 \mathrm{~cm}$. After the discharge, the flaky and reticulated SWNTs (AP-SWNTs) were collected in the arc chamber.

\section{The purification of SWNTs}

AP-SWNTs were purified using the traditional two-step method which described in literature [9]. First, AP-SWNTs were burned in the air at high temperature. A certain mass of AP-SWNTs were measured in a crucible, and the crucible was placed in a resistance furnace and roasted for $2 \mathrm{~h}$ at a temperature of $380{ }^{\circ} \mathrm{C}$. During this process, most amorphous carbon was removed, and SWNTs (APU-SWNTs) burned were obtained. Then APU-SWNTs were treated with $\mathrm{HCl}\left(12 \mathrm{~mol} / \mathrm{L}^{-1}\right)$ to acidify, the process is as follows: APU-SWNTs $(150 \mathrm{mg})$ were put in $250 \mathrm{ml}$ round bottom flask, and $\mathrm{HCl}(150 \mathrm{ml}, 12 \mathrm{~mol} / \mathrm{L})$ were added, the reaction system was reflow under the condition of $50{ }^{\circ} \mathrm{C}$ for $24 \mathrm{~h}$, the upper yellow solution was poured out after cooling, then the samples were filtered with $\operatorname{PTFE}(0.22 \mathrm{~mm})$, at the same time deionized water was used to wash the samples on the membrane filter for many times, until the $\mathrm{pH}$ of cleaning solution is neutral, in the process, most of the metal catalyst was got rid of, SWNTs after acidification with hydrochloric acid(HClSWNTs) were obtained. Finally Dimethyl sulfoxide (DMSO) was used to dispersed HCl-SWNTs under ultrasonic, yellow solution was poured out, DMSO was continued to added for ultrasonic dispersion, until the supernatant liquid was colorless, in this process, carbon impurity in the sample which cab be soluble in DMSO was got rid of, the high purity water insoluble sample(DMSO/HCl-SWNTs) was got.

\section{RESULTS AND ANALYSIS The preparation of SWNTs}

\section{The effect of helium pressure on the yield of AP- SWNTs}

As is shown in table 1 , the mass of APSWNTs varies with the pressure of helium; it means the output of AP-SWNTs is related to the pressure of helium, when the pressure of helium is $480 \mathrm{~T}$, the output of AP-SWNTs reached its peaks.

Table-1: The effect of helium pressure on the yield of AP-SWNTs

\begin{tabular}{|l|l|l|l|l|l|}
\hline The pressure of helium & $450 \mathrm{~T}$ & $\mathbf{4 6 0} \mathrm{T}$ & $\mathbf{4 7 0} \mathrm{T}$ & $\mathbf{4 8 0} \mathrm{T}$ & $\mathbf{4 9 0} \mathrm{T}$ \\
\hline The quality of the AP-SWNTs & $2.42 \mathrm{~g}$ & $2.45 \mathrm{~g}$ & $2.49 \mathrm{~g}$ & $2.58 \mathrm{~g}$ & $2.49 \mathrm{~g}$ \\
\hline
\end{tabular}

\section{The effect of current on the yield of AP-SWNTs}

As is shown in table 2 , the mass of AP-

SWNTs varies with the current, It means the output of
AP-SWNTs is related to the current, when the current is $100 \mathrm{~A}$, the output of AP-SWNTs reached its peaks.

Table-2: The effect of current on the yield of AP-SWNTs

\begin{tabular}{|l|l|l|l|l|l|}
\hline The current & $\mathbf{7 0} \mathbf{A}$ & $\mathbf{8 0} \mathbf{A}$ & $\mathbf{9 0} \mathbf{A}$ & $\mathbf{1 0 0} \mathbf{A}$ & $\mathbf{1 1 0} \mathbf{A}$ \\
\hline The quality of the AP-SWNTs & $2.21 \mathrm{~g}$ & $2.29 \mathrm{~g}$ & $2.37 \mathrm{~g}$ & $2.62 \mathrm{~g}$ & $2.60 \mathrm{~g}$ \\
\hline
\end{tabular}




\section{The purification of SWNTs} The SEM images of SWNTs

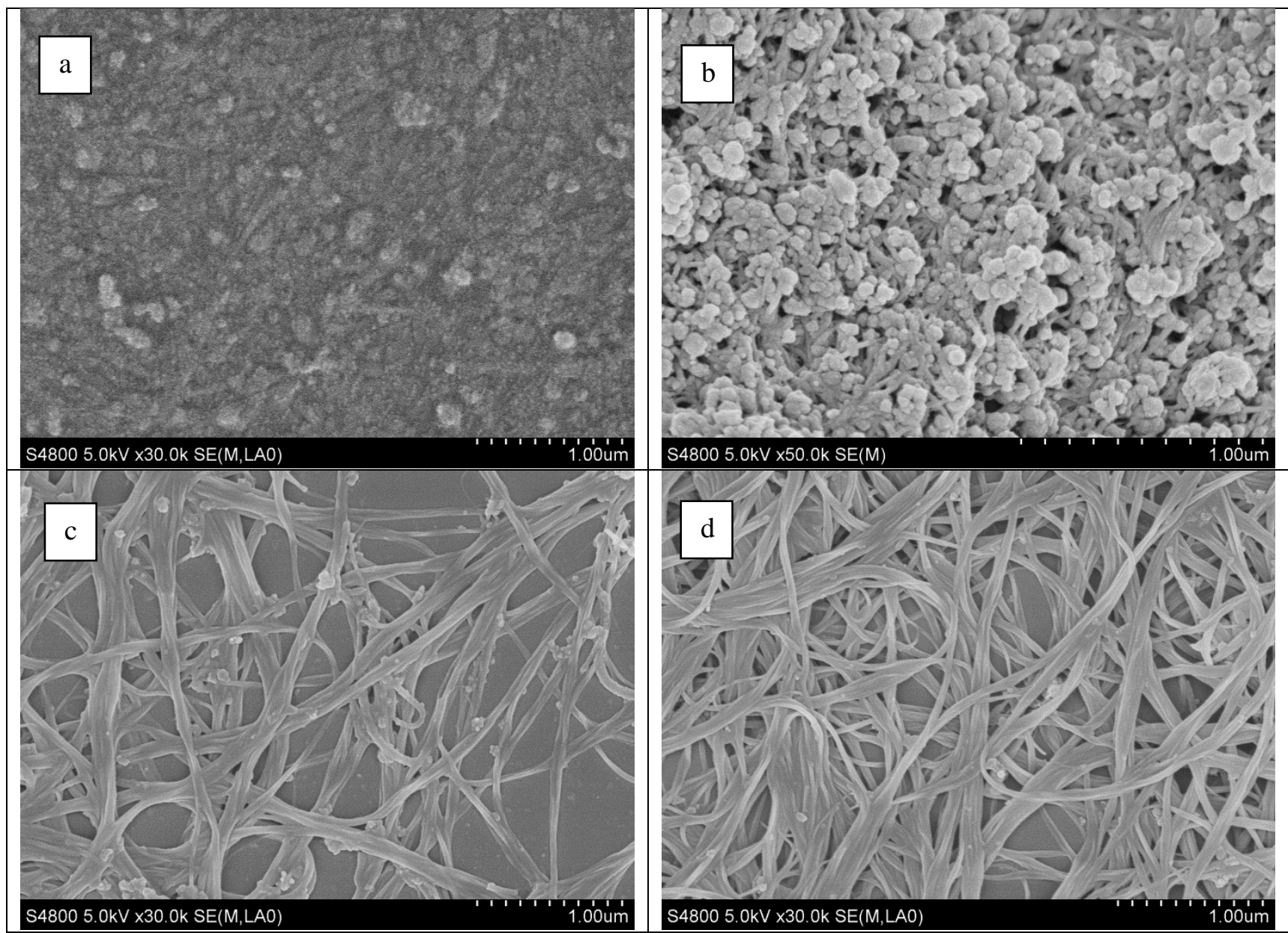

Fig-1: SEM images of (a) AP-SWNTs, (b) APU-SWNTs, (c) HCl-SWNTs, and (d) DMSO-SWNTs

The morphology of SWNTs can be observed by high-resolution scanning electron microscopy (SEM). Figure 1 are the SEM images of SWNTs processed by different methods, the figure (a) is the SEM image of AP-SWNTs. It can be seen that the whole image is very fuzzy with SWNTs bundles distributed among a large number of metal catalysts and carbon particles. The figure (b) is the SEM image of APU-SWNTs, which can be seen in figure (b), it is clearer than figure (a), and a large number of SWNTs bundles can be seen, indicating that a large number of metal catalysts were effectively removed from the samples after burning. Figure (c) is the SEM image of HCl-SWNTs. It can be seen that the tube bundles of SWNTs in figure (c), it is more clearly visible than those in figure (b), indicating that some carbon impurities were acidified with hydrochloric acid. Figure (d) is the SEM images of DMSO-SWNTs, comparing with (c), figure (d) is more clearly, carbon particles were further removed after treatment with organic solvent DMSO. So the samples including catalyst metal particles, most of the impurities, such as amorphous carbon and graphite particles. From the perspective of purification, SWNTs samples prepared by arc discharge with high purity were obtained after the above gas phase heat treatment, liquid phase acid treatment, selective dissolution of liquid phase organic solvent.

\section{The Vis-NIR spectra of SWNTs}

Vis-NIR spectroscopy is an important method to characterize the purity of SWNTs samples. The characteristic peaks of SWNTs prepared by dc arc discharge method are as follows: $\mathrm{S}_{22}$ peaks $(\sim 1020 \mathrm{~nm})$, $\mathrm{S}_{33}$ peaks $(\sim 500 \mathrm{~nm})$ and $\mathrm{S}_{44}$ peaks $(\sim 450 \mathrm{~nm})$ of $\mathrm{s}-$ SWNTs, which are generated by the electronic transition of the four interbands of s-SWNTs. The $\mathrm{M}_{11}$ peak of $\mathrm{m}$-SWNTs $(\sim 700 \mathrm{~nm})$ is due to the electron transition in its first band.

Accordingly, the SWNTs samples were subjected to analyse by Vis-NIR spectroscopy and the analysis data were normalized. As shown in figure 2, the first $\mathrm{M}_{11}$ peak with electron transition of $\mathrm{m}$-SWNTs occurred between 600 and $800 \mathrm{~nm}$, the $S_{22}$ peak of sSWNTs occurred between 800 and $1200 \mathrm{~nm}$, and the $\mathrm{S}_{33}$ and $\mathrm{S}_{44}$ peak of s-SWNTs occurred between 400 and $600 \mathrm{~nm}$. Curve (a) is the absorption curve of APSWNTs, there is not obvious peak in Curve (a). 
Curve(b) is the absorption curve of APU-SWNTs, there is one small absorption peak(1012 nm), compared with curve (a), it can be found that the peak area of curve(b) increased obviously, it instructed that most of the amorphous carbon impurities were removed after high temperature calcination, the purity of SWNTs samples was increased. Curve (c) is the absorption cuve of $\mathrm{HCl}-$ SWNTs, there is one small absorption peak (1014 nm), compared with curve (b), it can be found that the peak area of curve(c) increased obviously, it instructed that most of the metal catalyst impurities were removed after the acidification of hydrochloric acid, the purity of SWNTs samples was increased and the absorption peak was blued at $2 \mathrm{~nm}$, further indicating that the dispersibility of SWNTs samples was improved. This is due to the van der Waals force between SWNTs bundles, as the impurities in SWNTs became less, their purity became higher, resulting in larger bundles of SWNTs [10]. Curve(d) is the absorption curve of DMSO-SWNTs, there is one small absorption peak $(1017 \mathrm{~nm})$, Compared with curve (c), it can be found that the peak area of curve(d) increased obviously, it instructed that the SWNTs sample were treated after DMF, carbon impurities were dissolved further in DMF, the purity of SWNTs samples was further improved. High purity SWNTs samples were obtained.

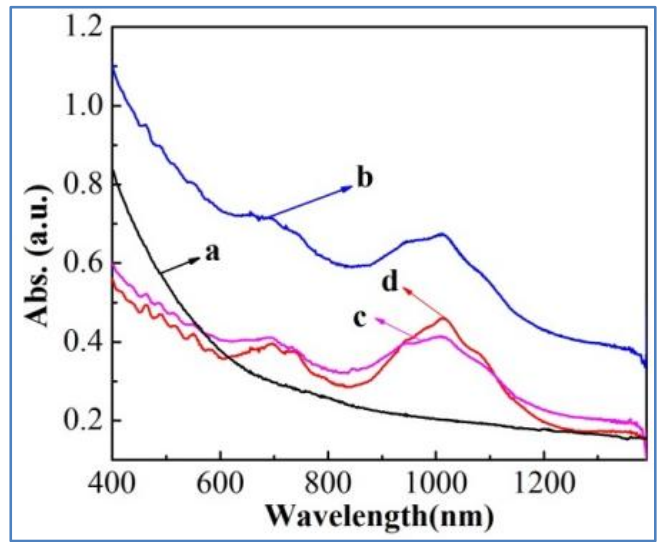

Fig-2: Vis-NIR spectra of (a) AP-SWNTs, (b) APU-SWNTs, (c) HCl-SWNTs, and (d) DMSO-SWNTs

The Raman spectra of SWNTs

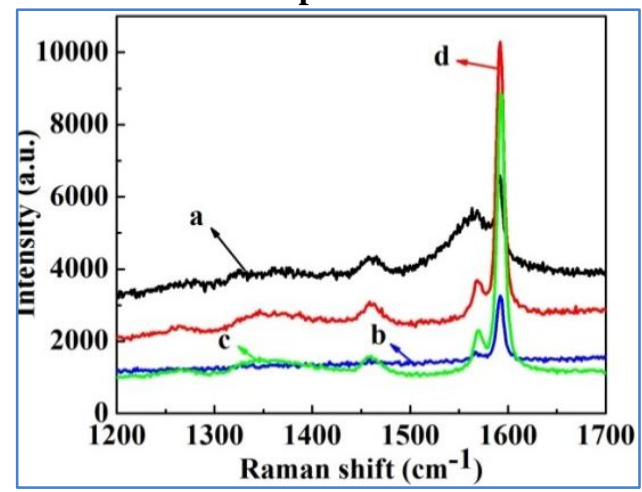

Fig-3: Raman spectra of (a) AP-SWNTs, (b) APU-SWNTs, (c) HCl-SWNTs, and (d) DMSO-SWNTs at $1200 \sim 1700 \mathrm{~cm}^{-1}$
Resonance Raman spectroscopy is another important method for the characterization of SWNTs samples [11]. The resonance Raman spectrum of SWNTs samples is located at D mode in 1330 1360 $\mathrm{cm}^{-1}$ and $\mathrm{G}$ peak in $1570 \sim 1600 \mathrm{~cm}^{-1}$, where the ratio of $G$ peak to $D$ peak intensity is related to the degree of purification of SWNTs. Accordingly, under the condition of excitation wavelength of $633 \mathrm{~nm}$, as is shown in figure 3 , as the purification proceeds, it is found that the $I_{G} / I_{D}$ is increased continuously, the $I_{G} / I_{D}$ of the curve a (AP-SWNTs) is 6.1 , the $\mathrm{I}_{\mathrm{G}} / \mathrm{I}_{\mathrm{D}}$ of the curve $b$ (APU-SWNTs) is 9.3 , the $\mathrm{I}_{\mathrm{G}} / \mathrm{I}_{\mathrm{D}}$ of the curve $\mathrm{c}$ (HCl-SWNTs) is 10.6 , the $\mathrm{I}_{\mathrm{G}} / \mathrm{I}_{\mathrm{D}}$ of the curve $\mathrm{d}$ (DMSOSWNTs) is 11.9. From the perspective of purification, SWNTs prepared by arc discharge with high purity were obtained after the above gas phase heat treatment, liquid phase acid treatment; selective dissolution of liquid phase organic solvent. This is consistent with SEM characterization before and after purification.

\section{CONCLUSION}

SWNTs were prepared by arc discharge. After burning, acidification and selective dissolution in organic solvents, SWNTs samples with high purity were obtained. During the purification of SWNTs, in addition to conventional air burning and acidification, selective dissolution of SWNTs in DMSO was also attempted, and the purity of SWNTs samples soaked in organic solvent DMSO was further improved. It can be said that this method provides another way of thinking for the purification of SWNTs. Some organic solvents can be selected to conduct ultrasonic treatment on SWNTs samples, so that some carbon impurities can be dissolved in the organic solvent to achieve the purpose of purifying SWNTs. Further purification of SWNTs samples with other organic solvents will be attempted to investigate the purification of SWNTs.

\section{ACKNOWLEDGMENTS}

This work was supported by the guiding science and technology plan project of Daqing (zd2019-44), the basic cultivation funding of Heilongjiang Bayi Agricultural University (ZRCPY201818), the doctoral research foundation of Heilongjiang Bayi Agricultural University (XDB201815).

\section{REFERENCES}

1. Morais, M. V., Olivaaviles, A. I., Matos, M. A., Tagarielli, V. L., Pinho, S. T., Hubner, C., \& Henning, F. (2019). On the effect of electric field application during the curing process on the electrical conductivity of single-walled carbon nanotubes-epoxy composites. Carbon: 153-167.

2. Xu, X., Zhai, J., Li, L., Chen, Y., Yu, Y., Zhang, M., \& Tang, Z. (2015). Passively mode-locking erbium-doped fiber lasers with $0.3 \mathrm{~nm}$ singlewalled carbon nanotubes.. Scientific Reports, 4(1):6761-6761.

3. Cao Q, \& Rogers J A. (2009). Ultrathin Films of 
Single- Walled Carbon Nanotubes for Electronics and Sensors: A Review of Fundamental and Applied Aspects [J]. Advanced Materials, 21(1): 29-53.

4. Lefebvre, J., Ding, J., Li, Z., Finnie, P., Lopinski, G. P., \& Malenfant, P. R. (2017). High-Purity Semiconducting Single-Walled Carbon Nanotubes: A Key Enabling Material in Emerging Electronics. Accounts of Chemical Research, 50(10): 24792486.

5. Feazell, R. P., Nakayamaratchford, N., Dai, H., \& Lippard, S. J. (2007). Soluble single-walled carbon nanotubes as longboat delivery systems for platinum (IV) anticancer drug design. Journal of the American Chemical Society, 129(27): 84388439.

6. Eatemadi, A., Daraee, H., Karimkhanloo, H., Kouhi, M., Zarghami, N., Akbarzadeh, A., \& Joo, S. W. (2014). Carbon nanotubes: properties, synthesis, purification, and medical applications. Nanoscale Research Letters, 9(1): 393-393.

7. Nakano, M., Nonoguchi, Y., Nakashima, T., Hata, K., \& Kawai, T. (2016). Solid-state, individual dispersion of single-walled carbon nanotubes in ionic liquid-derived polymers and its impact on thermoelectric properties. RSC Advances, 6(3): 2489-2495.

8. Yuan, X., An, Y. L., Zhang, C., \& Sui, H. C. (2012). Preparation of Single-Walled Carbon Nanotubes from Starch by Arc Discharge. Advanced Materials Research: 63-66.

9. Bertoncini, M., Coelho, L. A., Maciel, I. O., \& Pezzin, S. H. (2011). Purification of Single-Wall carbon nanotubes by heat treatment and supercritical extraction. Materials Research-iberoamerican Journal of Materials, 14(3): 380-383.

10. Azevedo, D. L., Sato, F., Filho, A. G., \& Galvao, D. S. (2011). van der Waals potential barrier for cobaltocene encapsulation into single-walled carbon nanotubes: classical molecular dynamics and ab initio study. Molecular Simulation, 37(9): 746-751.

11. Nagy, P., Koltai, J., Surjan, P. R., Kurti, J., \& Szabados, A. (2016). Resonance Raman Optical Activity of Single Walled Chiral Carbon Nanotubes. Journal of Physical Chemistry A, 120(28): 5527-5538. 